\title{
Investigating the Effectiveness of Fear Appeals to Prompt Help Seeking in At-Risk Gamblers: The Moderating Role of Problem Gambling Status: An Abstract
}

\author{
Svetlana De Vos, Roberta Crouch, Pascale Quester, and Jasmina Ilicic
}

\begin{abstract}
This research examines the moderating role of problem gambling status (low-risk gamblers, moderate-risk gamblers and problem gamblers) on the processing of fear appeals in a sample of Australian gamblers.

This study uses multigroup comparisons in structural equation modelling (SEM) to test the robustness of the revised protection motivation model (RPMM) in predicting the effectiveness of fear appeals to induce help-seeking intentions in at-risk gamblers. The results provide evidence that fear does not mediate the impact of perceived susceptibility on help-seeking intentions in at-risk gamblers. Cognitive appraisals of perceived susceptibility directly and positively impact help-seeking intentions in low, moderate and problem gamblers.

Overall, this research demonstrates that negative emotion such as fear does not prompt at-risk gamblers to seek help. However, when gambling threat is perceived as relevant, the cognitive appraisals of gambling risks predict significant helpseeking intentions in low, moderate and problem gamblers. Direct impact of perceived susceptibility on help-seeking intentions does not significantly differ among low, moderate and problem gamblers. This research demonstrates the unique role of individual gambling status on fear appeal effectiveness in at-risk gamblers.
\end{abstract}

\footnotetext{
S. De Vos $(\varangle)$

Australian Institute of Business, Adelaide, Australia

University of Adelaide, Adelaide, Australia

e-mail: svetlana.devos@adelaide.edu.au

R. Crouch • P. Quester

University of Adelaide, Adelaide, Australia

e-mail: roberta.crouch@adelaide.edu.au; pascale.quester@adelaide.edu.au

J. Ilicic

Monash Business School, Monash University, Melbourne, Australia

e-mail: jasmina.ilicic@monash.edu
} 